\title{
Food-based dietary guidelines: some assumptions tested for the Netherlands
}

\author{
M. R. H. Löwik*, K. F. A. M. Hulshof and J. H. Brussaard \\ Department of Consumer Research and Epidemiology, TNO Nutrition and Food Research Institute, PO Box 360, 3700 AJ, \\ Zeist, The Netherlands
}

\begin{abstract}
Recently, the concept of food-based dietary guidelines has been introduced by WHO and FAO. For this concept, several assumptions were necessary. The validity and potential consequences of some of these assumptions are discussed in this paper on the basis of the Dutch National Food Consumption Surveys. The topics were interrelationships among dietary characteristics, nutrient density, diets in accordance with the guidelines, and food variety.

To obtain insight into overall diet quality, a dietary quality index based on nutrients and a foodbased quality index based on food groups were created and tested. As expected the results showed that a higher dietary quality index was associated with an improved intake of the nutrients included in the index, in particular a lower intake of total fat and cholesterol. This was related to a lower consumption of cheese, fats and oils, meat and meat products, and a higher consumption of fruit and vegetables. The information obtained with a dietary quality index can be used to assess the feasibility of a particular goal in combination with other relevant goals and to obtain clues or confirmation for recommendations regarding food consumption.

The food-based quality index created showed that a combination of several food-based goals (formulated in quantitative terms) was associated with an increase in food consumption, without a clear relevance for the dietary quality. Therefore, the formulation of food-based guidelines should be based on explicitly stated dietary goals. The findings regarding nutrient density were in the direction of what was assumed, namely a positive association between density and absolute intake, although the expected effects were not completely found. The results regarding the association between variety and dietary intake were characterized mainly by differences in quantity rather than dietary quality.

The data indicate that energy intake may be an important pitfall in implementing food-based dietary guidelines, that 'eat a variety of food' can be an empty slogan and that nutrient density is positively related to the absolute intake of specific micronutrients. Further, the 'absence' of interrelationships among risk nutrients indicates that a selection process has to be applied.
\end{abstract}

Food-based dietary guidelines: The Netherlands

According to Hegsted (1986), dietary standards must ultimately be translated into food products keeping in mind the nature of food supply and current dietary practices. In line with this, the concept of food-based dietary guidelines (FBDG) has been proposed by WHO/FAO (1996).

Dietary advice can be given based on knowledge of the main nutritional risk areas and insight into the main sources of nutrients and bioactive dietary components (Löwik, 1993); the nutritional risk areas are identified through the differences between dietary standards and prevailing intake levels and nutritional status. The sources of energy and nutrients provide target foods whose consumption can be changed to improve the diet on a population basis. The identification of the major sources of energy and nutrients in population groups is mostly based on the relative contribution of the various groups of food products to daily intake (Welsh \& Marston, 1982; Block et al. 1985; Cook \& Welsh, 1987), which takes into account number of consumers, frequency of consumption, quantity consumed and nutrient content in food products. To be effective in nutrition education of the general public, the food items have to be classified into relatively homogeneous groups, which fit in with consumers' perception of logical classification (Southgate, 1992). Furthermore, an effective strategy requires a selection process with priority setting, because the message has to be as simple as possible. If all dietary goals and 
recommendations have to be achieved at the same time, a large multi-criteria decision model has to be established to guide policy processes and consumer behaviour.

Although the concept of food-based dietary guidelines is attractive, the validity and consequences of the assumptions are not yet tested. The major assumptions studied in this paper are:

- Changing the diet into the direction of one dietary goal will have a favourable effect on other goals as well.

- A higher nutrient density is associated with a higher intake of the particular nutrient.

- A higher food variety is associated with an improved quality of diet.

- The implementation of a dietary quality index and/or food-based guidelines will result in an improved quality of diet.

This paper starts with a selection process in that the main risk nutrients in the Dutch population are identified. Since fat consumption is given highest priority, information is provided on differences in dietary intake according to different levels of fat consumption. Subsequently, the interrelations of fat consumption with other risk nutrients are presented in order to assess potential (un)favourable sideeffects of a reduced fat intake. Special attention is given to two elements of food-based dietary guidelines, namely nutrient density and food variety. Finally, several aspects are integrated in that the effects of overall diet quality indices (one based on nutrients and the other on food groups) are studied. The results presented are based on the first and the second Dutch National Food Consumption Survey (DNFCS).

The first Dutch National Food Consumption Survey (DNFCS) was conducted in 1987-1988, and the second survey in 1992 (Löwik et al. 1994, 1998). Both surveys were conducted within the framework of the Dutch Nutrition Surveillance System (Löwik \& Hermus, 1988). Data were obtained from a probability sample of non-institutionalized subjects. Information on food consumption was collected by means of $2 \mathrm{~d}$ records. Methods regarding sampling procedure and dietary data collection have been published in detail (Hulshof \& van Staveren, 1991).

\section{Risk nutrients and groups}

Implementation of the concept of food-based dietary guidelines starts with the identification of prevailing nutritional problems. In the Netherlands, the intake of fat, especially saturated fatty acids (SFA) is high, whereas the intakes of carbohydrates (especially polysaccharides) and dietary fibre (relative to energy intake) are low. For micronutrients, mean intake of vitamin B-6 per g protein, iron (in boys aged 1-3 and 13-18, girls aged 1-3 and women aged 10-49) and vitamin A (in boys aged 10-12, girls aged 7-12 and pregnant women) are below recommended dietary allowances. Overweight individuals, a low intake of iodine and a high sodium intake are also prevalent in the Dutch population. In a Dutch survey on the nutritional status of people 20-80 years old, with special reference to micronutrients, it was found that the group most at risk for suboptimal micronutrient supply was women aged 20-49 (vitamin B-6, iron, iodine, and folate for those who wished to become pregnant). These risks could be modified favourably by lifestyle changes (Brussaard et al. 1997). Based on this observation, it is reasonable to examine the formulation of food-based dietary guidelines for the Netherlands for women 20-49 years old and to investigate potential unfavourable sideeffects in other population groups. Risks associated with inadequacy of intakes of vitamin B-6, iron and vitamin A are still in the phase of problem formulation. Policy processes for sodium are currently at an intermediate stage (Löwik et al. 1996). For fat intake (and hence, to a certain extent, indirectly intake of carbohydrate and dietary fibre) and iodine intake the policy processes are beyond the phase of problem formulation, in that actions to improve the situation have been or are being implemented.

In the late 1980s and the early 1990s, fat intake, especially the intake of SFA, was given the strongest emphasis in several policy statements because scientific evidence for its effect on health is strongest and the impact of a change in fat intake on public health is likely to be greatest (Löwik et al. 1996, 1997).

\section{Fat intake}

Since a reduction in fat consumption ranks high on the nutritional policy agenda in the Netherlands, it is of interest to obtain information on differences in nutrient intakes at different fat intake levels. Selected results, after a classification based on total fat as a percentage of total energy intake (\%energy, tertiles), among people 18-60 years old in the second DNFCS are presented in Table 1. A higher total fat intake (\%energy) was associated with a higher intake of energy and vitamin $\mathrm{A}$, a lower intake of vitamin $\mathrm{C}$ and a lower nutrient density (results not shown). In the highest tertile more energy was derived from fat (by definition) and less from protein, alcohol and, especially, carbohydrates. Major differences as to food groups were found for potatoes, cheese, nuts, seeds and snacks, fats and oils, and meat and meat products (higher mean values in the highest tertile), whereas higher fat intake was associated with a lower mean consumption of alcoholic beverages, fruit and, to a lesser extent, vegetables. The results should be interpreted, however, with care since the data refer to $2 \mathrm{~d}$ records in a cross-sectional design.

\section{Interrelationships}

Ideally, a single dietary variable would be available as the indicator of all nutritional problems identified in that a change in this variable in the favourable direction would result in a positive change in all other relevant dietary characteristics. As indicated above, a reduction of (saturated) fat intake was the primary target of nutritional policy in the Netherlands. Table 2 shows the interrelations (correlations) with other dietary factors. The results show that, in a cross-sectional design, a lower intake of SFA (\%energy) is accompanied by a lower intake of monounsaturated fatty acids (MUFA) (\%energy) and vitamin A, and a higher intake (\%energy) of polysaccharides, total carbohydrates and alcohol as well as (absolute) vitamin B-6. Weak associations $(r<0.10)$ were found for intakes of protein 
Table 1. Daily intake (mean \pm SD) of energy and nutrients among respondents $18-60$ years old in the second DNFCS according to fat intake

\begin{tabular}{lccc}
\hline & \multicolumn{3}{c}{ Total fat intake (\%energy) } \\
\cline { 2 - 4 } & Lowest tertile & Intermediate tertile & Highest tertile \\
\hline Energy (MJ) & $9 \cdot 1 \pm 3 \cdot 1$ & $9 \cdot 7 \pm 2 \cdot 8$ & $10 \cdot 2 \pm 3 \cdot 1$ \\
Protein (\%energy) & $16 \cdot 2 \pm 4 \cdot 4$ & $15 \cdot 2 \pm 3 \cdot 2$ & $14 \cdot 6 \pm 2 \cdot 9$ \\
Fat (\%energy) & $30 \cdot 1 \pm 4 \cdot 1$ & $37 \cdot 5 \pm 1 \cdot 6$ & $44 \cdot 9 \pm 3 \cdot 8$ \\
Carbohydrates (\%energy) & $48 \cdot 4 \pm 7 \cdot 5$ & $44 \cdot 0 \pm 5 \cdot 4$ & $38 \cdot 4 \pm 5 \cdot 5$ \\
Alcohol (\%energy) & $5 \cdot 4 \pm 7 \cdot 3$ & $3 \cdot 3 \pm 4 \cdot 6$ & $2 \cdot 2 \pm 3 \cdot 6$ \\
Iron (mg) & $12 \cdot 3 \pm 4 \cdot 4$ & $12 \cdot 7 \pm 4 \cdot 2$ & $12 \cdot 4 \pm 4 \cdot 1$ \\
Vitamin A (mg) & $0 \cdot 68 \pm 1 \cdot 03$ & $0 \cdot 75 \pm 0 \cdot 85$ & $0 \cdot 82 \pm 1 \cdot 04$ \\
Vitamin B-6 ( $\mu$ g/g protein) & $20 \pm 6$ & $19 \pm 5$ & $19 \pm 6$ \\
Vitamin C (mg) & $80 \pm 58$ & $75 \pm 50$ & $65 \pm 43$ \\
Potatoes (g) & $103 \pm 93$ & $121 \pm 98$ & $142 \pm 108$ \\
Alcoholic beverages (g) & $319 \pm 559$ & $185 \pm 324$ & $134 \pm 267$ \\
Fruit (g) & $133 \pm 135$ & $107 \pm 113$ & $90 \pm 104$ \\
Vegetables (g) & $147 \pm 106$ & $144 \pm 96$ & $128 \pm 91$ \\
Cheese (g) & $27 \pm 26$ & $34 \pm 32$ & $38 \pm 35$ \\
Nuts, seeds and snacks (g) & $20 \pm 33$ & $28 \pm 37$ & $42 \pm 51$ \\
Fats and oils (g) & $41 \pm 37$ & $50 \pm 36$ & $62 \pm 37$ \\
Meat and meat products $(\mathrm{g})$ & $111 \pm 32$ & $119 \pm 65$ & $128 \pm 72$ \\
\hline
\end{tabular}

(\%energy), polyunsaturated fatty acids (PUFA) (\%energy) and iron, and consumption of fruit and vegetables (as one combined group) with total and saturated fat. Comparable results were found in the various age-sex groups (results not shown). These results suggest that the assumption that, except for carbohydrates, other relevant dietary intake variables will change in a favourable direction (or at least stay the same) when (saturated) fat intake is lowered is not a valid assumption. The interpretation of the results is hampered by the cross-sectional design of the data.

For an integrated approach, several targets have to be taken into account. However, this will have a negative impact on the communication message and will demand unrealistically much of the target groups in terms of time, interest and knowledge. For instance, it is not realistic to assume that consumers can select food products on the basis of (sometimes even conflicting) nutritional criteria. These considerations call for priority setting and a temporary limitation of the number of dietary goals (preferably just

Table 2. Correlation coefficients for the relationship between $\%$ dietary energy as total fat or SFA and intakes of selected nutrients and foods in the Dutch National Food Consumption Survey (1992)

\begin{tabular}{lcc}
\hline & $\begin{array}{c}\text { Total fat } \\
\text { (\%energy) }\end{array}$ & $\begin{array}{c}\text { Saturated fat } \\
\text { (\%energy) }\end{array}$ \\
\hline Protein (\%energy) & -0.15 & -0.04 \\
SFA (\%energy) & 0.79 & 1 \\
MUFA (\%energy) & 0.87 & 0.58 \\
PUFA (\%energy) & 0.57 & 0.09 \\
Polysaccharides (\%energy) & -0.24 & -0.3 \\
Carbohydrates (\%energy) & -0.7 & -0.58 \\
Alcohol (\%energy) & -0.15 & -0.13 \\
Vitamin A (mg/d) & 0.11 & 0.11 \\
Vitamin B-6 (mg/g protein) & -0.07 & -0.17 \\
Iron (mg/d) & 0.13 & 0.05 \\
Fruits and vegetables (g/d) & -0.09 & -0.04 \\
\hline
\end{tabular}

$\mathrm{SFA}=$ saturated fatty acids; MUFA = monounsaturated fatty acids; PUFA $=$ polyunsaturated fatty acids. one). The selection of goals may change as our knowledge of the linkage between diet and health increases and as a result of changes in dietary intake characteristics.

\section{Nutrient density}

Hegsted (1992) suggests the concept of nutrient density (amount per MJ) to be a good framework for dietary standards. Food-based dietary guidelines are in line with this notion, in that nutrient density is used to establish and evaluate food-based dietary guidelines (WHO/FAO, 1996). The underlying assumption is that if a diet meets the energy requirement of individuals, it will also satisfy recommended nutrient intakes for all essential nutrients. This approach permits the simplification of age- and sex-related recommended nutrient intakes figures since the values are supposed to differ minimally when expressed per unit of energy. Looking at the Dutch recommendations, the differences among population groups are indeed smaller for nutrient density than for absolute intake levels, but that the effect should not be overestimated.

For the US population, Windham et al. (1981) conclude that, on average, all age-sex groups after infancy have diets of similar composition or nutrient density. Data from the Netherlands (Löwik et al. 1994) do not confirm this conclusion entirely since sometimes substantial differences in mean values were found among the population groups studied. For instance, the lowest densities were found among young children for iron, vitamin A and thiamin and among older children and adolescents for riboflavin, vitamin B-6, vitamin C, calcium, phosphorus and potassium. Furthermore, an implicit assumption regarding the usage of nutrient densities is probably that there exists a positive relation between intake of nutrients and density of the diet. In the second DNFCS, absolute intakes were indeed lower at lower nutrient densities. In the total population of the second DNFCS, the correlations between absolute intake and density were 0.51 for iron, 0.61 for calcium, 0.93 for 
vitamin A, 0.81 for thiamin, 0.54 for riboflavin, 0.52 for vitamin B-6 and 0.83 for vitamin C. These correlations show positive relations that are, however, not complete.

Finally, taking the total dietary intake as starting point, it is of interest to look at combinations of low and high nutrient densities; this has been done by Van der Beek et al. (1994). The observations confirm the existence of a positive association between intake and density of micronutrients. This association is also accompanied by differences in energy intake in that a lower mean energy intake were associated with a higher intake and density of vitamins. The results indicate that the assumptions regarding nutrient density as incorporated in FBDG are valid as to the direction of the relations but that the effects are not complete.

\section{Food variety}

Eating a variety of foods is often one of the recommendations of guidelines to achieve dietary adequacy. A wide range of foods is recommended to assure adequate nutrient intake, to avoid deficiencies and excessively high intakes of single nutrients, and to reduce the likelihood of high exposure to food contaminants. According to WHO/FAO (1996), there is enough evidence to justify promoting food variety through FBDG as a technique to reduce morbidity and mortality while awaiting further scientific studies on how exactly it operates. Variety may refer to variation in foods selected from different major food groups, foods within these food groups and/or preparation methods, whereas the effects may differ across nutrients. Therefore, the influence of variety on intake of energy and nutrients in the Netherlands was studied with a comprehensive model in which different aspects of variety were taken into account simultaneously (Hulshof et al. 1993).

The results (Table 3) show that associations between variety and dietary intake were characterized mainly by differences in quantity and not in nutrient density.

The term 'food variety category' was based on 43 food categories. In total, 759 different food items (codes corresponding to the Dutch food composition table) were reported by the selected adults during the 2-day period. These food items were (a priori) classified into 43 food groups based on food origin and pattern of use. Subjects were rated according to the numbers of these categories consumed over the period. The lowest category represents

Table 3. Daily intake of energy and nutrients (mean \pm SD) among men aged 22-49 in the first DNFCS, according to category of food variety ${ }^{1}$

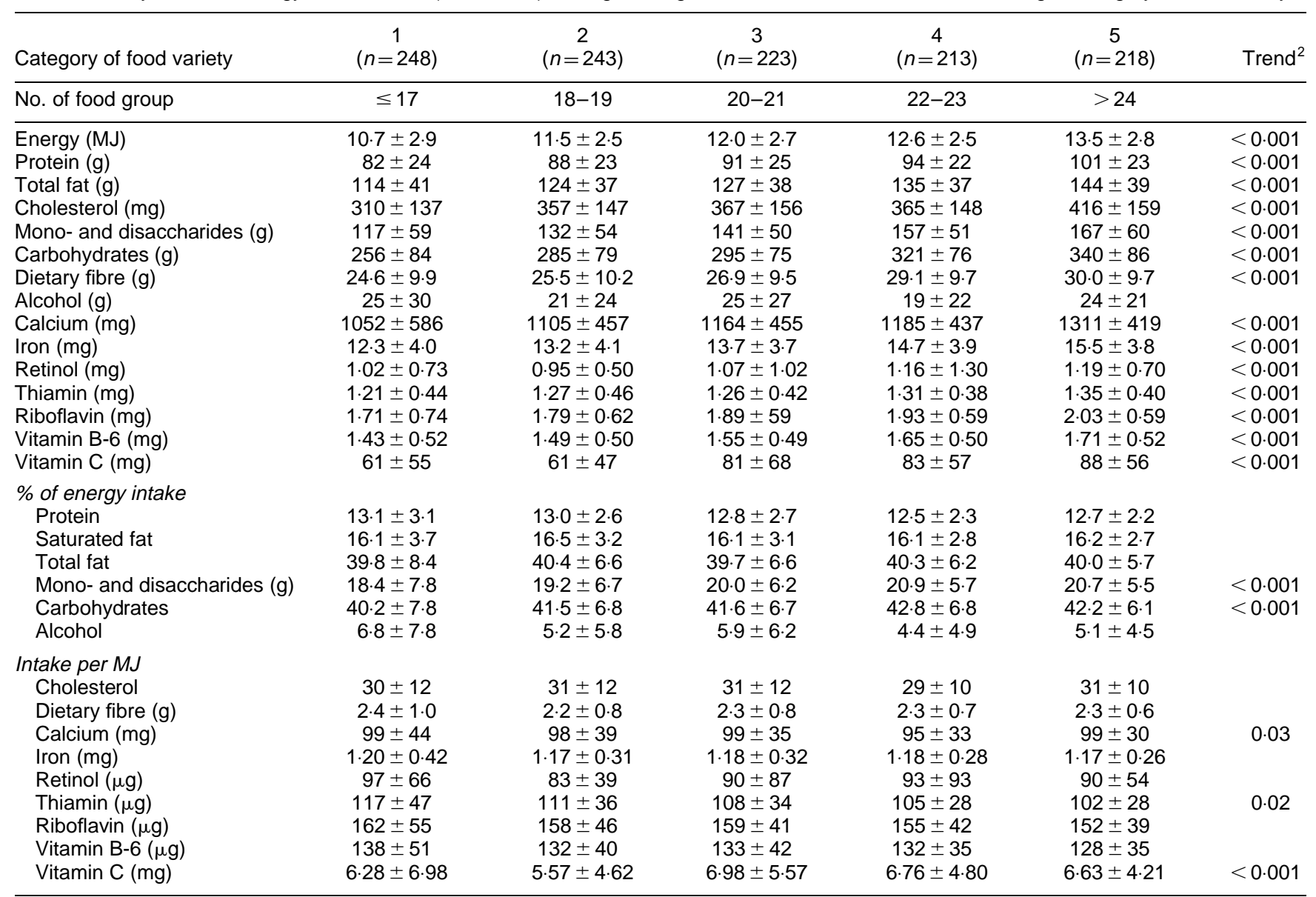

\footnotetext{
${ }^{1}$ Maximum is 43 food groups.

${ }^{2}$ Trend across categories.

$P$ values are presented.

Source: Hulshof et al. (1993).
} 
the least varied diet, while the highest category represents the most varied diet. For a more detailed description of the methods see Hulshof et al. (1993).

As to the impact of food groups to the variety in the diet, the inclusion of fruits and vegetables resulted in a higher variety score. Food variety was found to be positively associated with body height, education (men only) and socio-economic status. Inverse associations were found for body mass index (women only) and the proportions of smokers and breakfast skippers. Thus, food variety was positively associated with energy intake, but not with a higher prevalence of obesity in groups with a higher consumption level. These results suggest that subjects with a higher variety score may be more engaged in physical activities. This, in turn, might lead to a higher variety in food selection to cover the higher energy requirement. At a high consumption level, people might become bored of eating a lot of a few items only. Furthermore, the higher intake seen in high-variety groups might be a temporary state because the results derive from $2 \mathrm{~d}$ dietary records. None the less, these results challenge the concept that promoting variety of food choice does not necessarily alter patterns of nutrient intake.

\section{Dietary quality index}

An index of overall diet quality can be based on nutrients only. Such an index can include all nutrients but, for practical reasons, it is recommendable to select the most important nutrients, in this case those included in the Dutch dietary guidelines for a healthy diet. The criteria selected were a daily diet providing:

a) less than $35 \%$ energy from fat;

b) less than $10 \%$ energy from SFA;

c) less than $33 \mathrm{mg} / \mathrm{MJ}$ cholesterol; d) more than $50 \%$ energy from carbohydrates; and

e) less than $25 \%$ energy from mono- and disaccharides.

For every criterion, one point was given when attained, thus creating a 5-point scale, with 0 points corresponding to the lowest dietary quality and 5 points to the maximum quality. Intake of dietary fibre was not included because of methodological problems regarding assessment of food composition. Table 4 presents selected results for Dutch women aged 22-49. As expected, a higher dietary quality score corresponded with a higher proportion meeting the separate criteria of the index, mono- and disaccharides being an exception to this rule. The largest differences were seen for total fat and total carbohydrates; obviously, meeting the criterion for SFA was not easy. A higher dietary quality score was associated with a lower consumption of cheese, fats and oils, and meat and meat products, and a higher consumption of fruit and vegetables (results not shown). This was confirmed by the proportion of women meeting the criteria of the food-based guidelines (Table 4). To a certain extent, these results are promising with respect to the feasibility of realization of a much healthier diet through adequate selection from the current food supply, because the differences among the dietary quality score groups are modest. A higher dietary quality was associated with a higher prevalence of a prescribed diet, especially energy restriction, and a higher educational level. There was an inverse relation between the dietary quality score and energy intake.

Mean energy intake was $8.7 \mathrm{MJ} / \mathrm{d}$ among those with a score of $0-1$ and $7 \cdot 0 \mathrm{MJ} / \mathrm{d}$ among women with a score of $4-5$. This may cast some doubt on the results in that selective under-reporting of energy-rich products may have taken place. In line with this, Table 4 might suggest over-reporting of products of vegetable origin. The characteristics of the

Table 4. Proportion of women 22-49 years old meeting the various goals and criteria according to the dietary quality index

\begin{tabular}{|c|c|c|c|c|}
\hline \multirow[b]{2}{*}{ Dietary quality score } & \multicolumn{4}{|c|}{ Dietary quality score* } \\
\hline & $\begin{array}{c}0-1 \\
(n=392)\end{array}$ & $\begin{array}{c}2 \\
(n=704)\end{array}$ & $\begin{array}{c}3 \\
(n=256)\end{array}$ & $\begin{array}{c}4-5 \\
(n=141)\end{array}$ \\
\hline \multicolumn{5}{|l|}{$\%$ achieving guideline } \\
\hline Total fat & 3 & 13 & 92 & 100 \\
\hline Saturated fat & 0 & 1 & 11 & 65 \\
\hline Cholesterol & 19 & 91 & 91 & 96 \\
\hline Total carbohydrates & 1 & 7 & 52 & 81 \\
\hline Mono- \& disaccharides & 68 & 89 & 54 & 71 \\
\hline \multicolumn{5}{|l|}{ Food-based guidelines } \\
\hline Bread & 11 & 16 & 18 & 20 \\
\hline Potatoes & 36 & 54 & 42 & 49 \\
\hline Vegetables & 30 & 37 & 39 & 39 \\
\hline Fruit & 22 & 23 & 32 & 40 \\
\hline Milk and milk products & 39 & 42 & 46 & 43 \\
\hline Cheese & 62 & 68 & 63 & 45 \\
\hline Meat/fish/eggs & 77 & 64 & 50 & 45 \\
\hline \multicolumn{5}{|l|}{ Characteristics } \\
\hline Not on a diet & 92 & 90 & 86 & 82 \\
\hline Energy-restricted diet & 5 & 6 & 7 & 14 \\
\hline $\begin{array}{l}\text { Lifestyle rule } \\
\end{array}$ & 2 & 3 & 4 & 8 \\
\hline Higher level of education & 39 & 50 & 54 & 62 \\
\hline
\end{tabular}

* One point is given for each dietary quality criterium that was met. 
women fit in with more socially desirable reporting during the 2 days of the survey, in that more women have the motivation (therapeutic diet) and the knowledge (higher education) for such a reporting pattern. If selective reporting partly explains the differences, caution is called for in using the data for policy-making processes.

As expected, the results suggest that a combination of dietary goals results mostly in a more favourable diet for the separate goals included in the index. The most important information that can be obtained through analyses regarding a dietary quality index is related to the feasibility of a goal in combination with other goals and the (direction of the) food consumption associated with improvement in the dietary quality index. Both aspects are important for an efficient nutrition education strategy.

\section{Food-based dietary guidelines}

One rationale behind the concept of FBDG is that consumers think in terms of foods rather than nutrients (WHO/FAO, 1996). The most important factors influencing consumer acceptance are current consumption patterns, and specific food recommendations or examples incorporated in a set of guidelines (Stowers, 1992). This is one of the reasons that food-based guidelines have to be country-specific. For the Netherlands, recommended quantities of several basic food groups are formulated for children, adolescents and adults by the Netherlands Bureau for Nutrition Education. For adults the following recommendations for daily intake are given:

- bread (including breakfast cereals): 5-7 slices;
- potatoes (including rice, pasta and pulses): $3-5$ pieces;

- vegetables: 3-4 serving spoons;

- fruit: 2 pieces;

- milk and milk products: $2-3$ glasses;

- cheese: 1-2 slices; and

- meat, fish and eggs: about $115-130 \mathrm{~g}$ (or $100 \mathrm{~g}$ cooked).

One point was given to a subject when the quantity consumed was at least at the lowest recommended level. Thus a food-based quality index was created with a scale ranging from 0 to 7 . The higher the score, the better the quality of the diet was assumed to be.

Table 5 presents selected characteristics of three groups of Dutch women with different scores for food-based dietary quality. Similar observations were made for males (results not shown). Obviously, a higher score correlated with a higher proportion of the women meeting the separate goals. The data indicate that meeting the goals for bread, fruit and, to lesser extent, milk (products) were most difficult. A higher score was associated with a higher intake of energy and a higher nutrient density. Possibly except for protein and alcohol, relatively small differences were found for the contribution of macronutrients to daily energy intake.

Apparently, a combination of the selected foodbased guidelines (formulated in quantitative terms) results in a selection of women with a relatively high energy intake. Furthermore, the combination selected does not have an important selective effect on the \%energy of the macronutrients.

A selective effect was found for nutrient density, which can be explained by the fact that bread, potatoes, vegetables,

Table 5. Selected characteristics of women 22-49 years old meeting the various goals and criteria according to the food-based quality score index

\begin{tabular}{lccc}
\hline & \multicolumn{3}{c}{ Food-based quality score } \\
\cline { 2 - 4 } & $0-2$ & $3-4$ & $5-7$ \\
& $(n=575)$ & $(n=728)$ & $(n=190)$ \\
\hline Dietary intake (mean $\pm \mathrm{SD})$ & & & \\
Energy (MJ/d) & $7 \cdot 6 \pm 2 \cdot 4$ & $8 \cdot 7 \pm 2 \cdot 3$ & $9 \cdot 7 \pm 2 \cdot 4$ \\
Protein (\%energy) & $14 \cdot 8 \pm 4 \cdot 1$ & $16 \cdot 0 \pm 3 \cdot 8$ & $16 \cdot 7 \pm 3 \cdot 4$ \\
Fat (\%energy) & $37 \cdot 9 \pm 7 \cdot 8$ & $38 \cdot 1 \pm 7 \cdot 0$ & $36 \cdot 7 \pm 6 \cdot 5$ \\
Saturated fat (\%energy) & $14 \cdot 3 \pm 3 \cdot 6$ & $14 \cdot 6 \pm 3 \cdot 1$ & $14 \cdot 5 \pm 2 \cdot 9$ \\
Carbohydrates (\%energy) & $44 \cdot 4 \pm 8 \cdot 2$ & $43 \cdot 4 \pm 7 \cdot 2$ & $44 \cdot 7 \pm 6 \cdot 1$ \\
Mono- and disaccharides (\%energy) & $20 \cdot 8 \pm 8 \cdot 4$ & $20 \cdot 0 \pm 6 \cdot 7$ & $21 \cdot 0 \pm 5 \cdot 8$ \\
Alcohol (\%energy) & $2 \cdot 9 \pm 5 \cdot 3$ & $2 \cdot 6 \pm 4 \cdot 4$ & $2 \cdot 0 \pm 3 \cdot 6$ \\
Nutrient density (mean \pm SD) & & & \\
Calcium (mg/MJ) & $102 \pm 51$ & $119 \pm 47$ & $135 \pm 50$ \\
Iron (mg/MJ) & $1 \cdot 3 \pm 0 \cdot 5$ & $1 \cdot 4 \pm 0 \cdot 4$ & $1 \cdot 5 \pm 0 \cdot 5$ \\
Vitamin A ( $\mu$ g/MJ) & $76 \pm 100$ & $82 \pm 92$ & $91 \pm 147$ \\
Thiamin ( $\mu$ g/MJ) & $136 \pm 90$ & $148 \pm 94$ & $158 \pm 79$ \\
Riboflavin ( $\mu$ g/MJ) & $160 \pm 71$ & $178 \pm 62$ & $194 \pm 58$ \\
Vitamin B-6 ( $\mu \mathrm{g} / \mathrm{MJ})$ & $165 \pm 63$ & $182 \pm 56$ & $188 \pm 50$ \\
Vitamin C (mg/MJ) & $7 \cdot 7 \pm 8 \cdot 1$ & $10 \cdot 2 \pm 8 \cdot 3$ & $11 \cdot 7 \pm 7 \cdot 1$ \\
Food-based guidelines (\% meeting the goal) & & & \\
Bread & 6 & 17 & 39 \\
Potatoes & 22 & 56 & 86 \\
Vegetables & 9 & 44 & 83 \\
Fruit & 10 & 30 & 56 \\
Milk and milk products & 22 & 48 & 79 \\
Cheese & 43 & 72 & 91 \\
Meat/fish/eggs & 39 & & \\
\hline & & & \\
\hline
\end{tabular}


fruit, milk (products), cheese and meat/fish/eggs are relatively good sources of micronutrients (Löwik et al. 1994, 1998). Based on these observations, it can be stated that a combination of several food-based goals (formulated in quantitative terms) can result in an increase of the food consumption that does not have an important effect on the quality of the diet. This holds especially when no food groups are restricted by an upper consumption level.

Furthermore, a group like fats and oils was not included in the food-based guideline, whereas the consumption of such a group is expected to have a direct relationship with the intake of nutrients. Within groups like milk and milk products, all products were classified equal in that no distinction was made for, for instance, full-fat and skimmed milk. In conclusion, in formulating food-based guidelines explicitly stated dietary goals should be the basis of the selection and quantification of these guidelines.

\section{Conclusions}

The observed association between food variety and dietary intake was mainly characterized by differences in quantity (amounts) and to a lesser extent by differences in quality of the diet. Therefore, fulfilment of the guideline 'Eat a variety of food', corresponding with a greater number of different foods consumed, will not automatically imply a more adequate diet. As shown by the analyses regarding foodbased dietary guidelines and food variety, improvements in nutrient intakes were accompanied by a higher energy intake. This indicates that a higher energy intake enhances the chance of meeting the guidelines. This is in line with the statement by WHO/FAO that, if the intake of adolescents or adults is less than $8.4 \mathrm{MJ} / \mathrm{d}$, it will be difficult to meet their vitamin and mineral needs. In contrast, a low fat intake was correlated with a relatively low energy intake. For reasons related to feasibility and effectiveness, it is recommended to concentrate the attainment of the dietary guidelines on a strongly limited number of nutrients (preferably just one). The selection may change over time as a result of changes in scientific and political insights as well as in trends in food consumption. Since women aged 20-49 were at a high nutritional risk, this group can be used as the starting point for the formulation of food-based dietary guidelines. It may be expected that other population groups will also benefit from the guidelines formulated in this way. A combination of food-based guidelines (formulated in quantitative terms) and an overall quality index may result in a selection of subjects with a high energy intake, especially when no food groups are restricted by an upper consumption level.

\section{References}

Block G, Dresser AM, Hartman AM \& Carroll MD (1985) Nutrient sources in the American diet: quantitative data from the NHANES II survey. I. Vitamins and minerals. American Journal of Epidemiology 122, 13-26.

Brussaard JH, Brants HAM \& Löwik MRH (1997) Nutritional status among adults with special reference to micronutrients (Dutch Nutrition Surveillance System. European Journal of Clinical Nutrition 51 (Suppl. 3), S1-S66.

Cook DA \& Welsh SO (1987) The effect of enriched and fortified grain products on nutrient intake. Cereal Food World 32, 191196.

Hegsted DM (1986) Dietary standards - Guidelines for prevention of deficiency or prescription for total health? Journal of Nutrition 116, 478-481.

Hegsted DM (1992) Defining a nutritious diet: need for new dietary standards. Journal of the American College of Nutrition 211, 241-245.

Hulshof KFAM \& van Staveren WA (1991) The Dutch National Food Consumption Survey: design, methods and first results. Food Policy 16, 257-260.

Hulshof KFAM, Löwik MRH, Kistemaker C, ten Hoor F \& Hermus RJJ (1993) Is food variety conducive to a more adequate diet? In Assessment of variety, clustering and adequacy of eating patterns (Dutch National Food Consumption Survey). PhD thesis, University of Limburg (Maastricht), Netherlands.

Löwik MRH (1993) The role of information in food and nutrition policy making processes: a system analysis. In Food and Nutrition Policy in Europe pp 91-99. [DG van der Heij, MRH Löwik and Th Ockhuizen, editors] Wageningen: Pudoc.

Löwik MRH \& Hermus RJJ (1988) The Dutch nutrition surveillance system. Food Policy 13, 359-365.

Löwik MRH, Brussaard JH, Hulshof KFAM, Kistemaker C, Schaafsma G, Ockhuizen T \& Hermus RJJ (1994) Adequacy of the diet in the Netherlands in 1987-1988 (Dutch Nutrition Surveillance System). International Journal of Food Sciences and Nutrition 45 (Suppl. 1), S1-S62.

Löwik MRH, Hulshof KFAM, Brussaard JH \& Brants HAM (1996) Nutrition assessment and dietary guidelines: experience from the Dutch Nutrition Surveillance System. Proceedings of the Nutrition Society 55, 705-723.

Löwik MRH, Hulshof KFAM, Riedstra M, Brants HAM \& van Wechem SN (1997) High fat intake: policy implication for the Netherlands. In Implementing dietary guidelines for healthy eating pp 302-315 [V Wheelock, editor] London: Chapman \& Hall

Löwik MRH, Hulshof KFAM, van der Heijden LJM, Brussaard JH, Burema J, Kistemaker C \& de Vries PJF (1998) Changes in the diet in the Netherlands: 1987-1988 to 1992 (Dutch Nutrition Surveillance System). International Journal of Food Sciences and Nutrition 49 (Suppl. 1), S1-S64.

Southgate DAT (1992) Dietary advice: foods or nutrients. Proceedings of the Nutrition Society 51, 47-53.

Stowers SL (1992) Development of a culturally appropriate food guide for pregnant Caribbean immigrants in the United States. Journal of the American Dietary Association 92, 331-336.

Van der Beek EK, Löwik MRH, Hulshof KFAM \& Kistemaker C (1994) Combinations of low thiamin, riboflavin, vitamin B6 and vitamin C intake among Dutch adults (Dutch Nutrition Surveillance System). Journal of the American College of Nutrition 13, 383-391.

Welsh SO \& Marston RM (1982) Zinc levels of the US food supply: 1909-1980. Food Technology 36, 70-76.

WHO/FAO (1996) Preparation and use of food-based dietary guidelines. Nutrition Programme, Geneva: WHO.

Windham CT, Wyse BW, Hurst RL \& Hansen RG (1981) Consistency of nutrient consumption patterns in the United States. Journal of the American Dietetic Association 78, 587-595. 